\title{
Intron definition, exon definition and back-splicing revisited
}
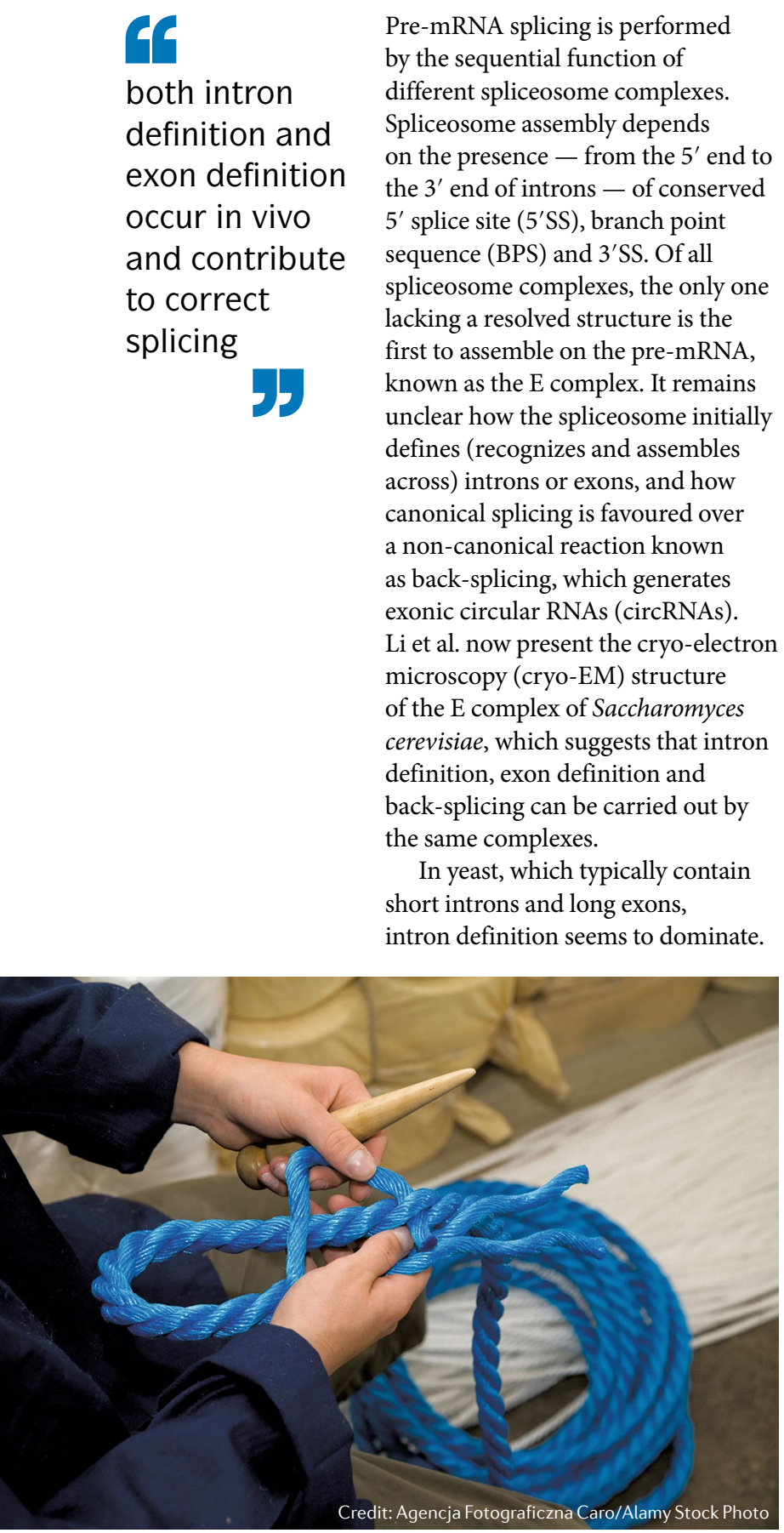

By contrast, in vertebrates, where introns are longer and exons are shorter, exon definition is thought to prevail. To study these mechanisms in detail, the authors assembled in vitro functional budding yeast $\mathrm{E}$ complexes on either the ACT1 pre-mRNA or the $U B C 4$ pre-mRNA, and determined their cryo-EM structures.

Key to initiating splicing through intron definition is bringing together the $5^{\prime}$ SS and the BPS. A striking feature found in the structure of the ACT1-E complex was a $\sim 25 \mathrm{bp}$ double helix downstream of the $5^{\prime} \mathrm{SS}$, which was not found in the UBC4-E complex. The 5'SS-to-BPS region (265 nt) of the $A C T 1$ intron, but not the same region in $U B C 4$ $(58 \mathrm{nt})$, is predicted to form long stem-like structures, and mutations that abolish these structures resulted in substantial pre-mRNA accumulation (splicing inhibition). Thus, secondary structures may help to bring together essential intronic elements and facilitate spliceosome assembly.

The structure of the E complex, especially the relative positions of the 5'SS and the BPS, suggested that the same E complex can form across exons: instead of connecting a 5'SS with a downstream BPS across an intron, the $5^{\prime} \mathrm{SS}$ could connect with an upstream BPS across an exon. To test whether exon definition occurs in vivo in yeast, the authors truncated the DYN2 gene to contain only its middle exon and flanking introns (IEI construct) and mutated the splicing elements bordering either side of the DYN2 exon - a BPS mutation in intron 1 and a $5^{\prime}$ SS mutation in intron 2. If splicing of DYN2 is governed solely by intron definition, retention of the intron in which a mutation resides would be expected, with minimal effect on the other intron; however, if splicing is governed by exon definition, each of the mutations would lead to the retention of both introns. The observed composition of splicing intermediates produced from the different IEI mutants suggested that both intron definition and exon definition occur in vivo and contribute to correct splicing of DYN2 and showed, for the first time, that exon definition occurs in yeast.

A prediction of this model is that formation of exon definition complexes across long exons leads to back-splicing - splicing of the $5^{\prime}$ SS downstream with the $3^{\prime}$ SS upstream of the exon - and the formation of exonic circRNAs, owing to the lack of steric hindrance. Shortening exons of circRNA-producing IEI constructs abolished circRNA formation from the constructs, supporting the preference for back-splicing across long (or multiple) exons and the occurrence of exon definition.

In summary, in yeast (and likely in all eukaryotes) the same E complexes are able to define both introns and exons, without the need for additional components or structural rearrangements. Furthermore, exon definition can cause back-splicing across long exons, suggesting that circRNAs are natural by-products of spliceosome-mediated splicing in all eukaryotes.

Eytan Zlotorynski

ORIGINAL ARTICLE Li, X. et al. A unified mechanism for intron and exon definition and back-splicing. Nature https://doi.org/10.1038/ s41586-019-1523-6 (2019)

RELATED ARTICLE Shi, Y. Mechanistic insights into precursor messenger RNA splicing by the spliceosome. Nat. Rev. Mol. Cell Biol. 18, 655-670 (2017) 